\title{
Exposition alimentaire aux mycotoxines cancérogènes dans le département de Séguéla (Nord-Ouest de la Cote d'Ivoire): cas de l'aflatoxine B1
}

\author{
Alimata FOFANA-DIOMANDE ${ }^{1 *}$, Konan Jean-Marie KOUAKOU², \\ Chantal AKA -DIEMELEOU ${ }^{2}$, Karim Sory TRAORE ${ }^{1}$ et Ardjouma DEMBELE ${ }^{2}$ \\ ${ }^{1}$ Laboratoire de Chimie, Santé et Environnement, UFR des Sciences et Gestion de l'Environnement, Université \\ Nangui Abrogoua, 02 BP 801 Abidjan 02, Côte d'Ivoire. \\ ${ }^{2}$ Laboratoire National d'Appui au Développement Agricole (LANADA), Laboratoire Central d'Agrochimie et \\ d'Ecotoxicologie (LCAE) 04 BP 612 Abidjan 04, Côte d'Ivoire. \\ *Auteur correspondant; E-mail: alimosyas@gmail.com ; 01 BP 2444 Abidjan 01, Côte d'Ivoire ;
} Tél: +22558170384

\section{RESUME}

Les aflatoxines sont des contaminants cancérogènes retrouvées fréquemment dans les denrées alimentaires. Dans cette étude, il s'agit de disposer d'informations relatives à la prévalence des aflatoxines dans les aliments de base du département de Séguéla. Pour ce faire, une identification des denrées alimentaires responsables de l'exposition des populations aux aflatoxines a été faite et le niveau de contamination de ces aliments en aflatoxines a été déterminé. Ainsi, une enquête basée sur le régime alimentaire a permis d'identifier le maïs, le riz, le manioc, l'arachide et le gombo comme les principales denrées alimentaires utilisées pour la confection des mets et de connaître les quantités journalières consommées par la population. L'échantillonnage réalisé selon la réglementation de la Commission Européenne (CE) 401/2006 et le guide LAB GTA 21 Révision 02/2017 du COFRAC a permis de déterminer leur charge en aflatoxines. Les résultats des analyses effectuées par HPLC avec extraction sur colonnes d'immuno-affinité, montrent des niveaux très variés de contamination des échantillons par l'Aflatoxine B1 (AFB1) allant de 0,02 à 18,65 $\mu \mathrm{g} / \mathrm{kg}$, avec 22\% des échantillons dépassant le seuil maximum de $2 \mu \mathrm{g} / \mathrm{kg}$ fixé en AFB1 par la CE $\mathrm{N}^{\circ} 1881 / 2006$. Ces teneurs obtenues en AFB1 conduisent à un risque d'exposition de la population aux effets toxiques de cette molécule. (C) 2019 International Formulae Group. All rights reserved

Mots clés: Denrées alimentaires, mycotoxines, aflatoxine B1, contamination, exposition.

\section{Food exposure to carcinogenic mycotoxins in the county of Séguéla (northwestern Ivory Coast): case of aflatoxin B1}

\begin{abstract}
Aflatoxins are carcinogenic contaminants frequently found in foodstuffs. In this study, we are going to get informations on the prevalence of aflatoxins in staple foods in the county of Séguéla. For that, an identification of the foodstuffs responsible for people exposure to aflatoxins has been made and the level of contamination of these foods with aflatoxins has been determined. Thus, a survey focussed on diet has allowed us to identify maize, rice, cassava, groundnuts and okra as the main foodstuffs used for making daily dishes.
\end{abstract}


This survey has also helpt to know the daily amounts of these foodstuffs consumed. The sampling carried out according to the European Commission (EC) 401/2006 regulation and the LAB GTA 21 Revision 02/2017 guide allowed to determine their content in aflatoxins. The results of the HPLC analyzes with extraction on immunoaffinity columns, show very varied levels of sample contamination by Aflatoxin B1 (AFB1) ranging from 0.02 to $18.65 \mu \mathrm{g} / \mathrm{kg}$, and $22 \%$ of samples exceeding the maximum threshold of $2 \mu \mathrm{g} / \mathrm{kg}$ set in AFB1 by EC No 1881/2006. These levels obtained in AFB1 lead to a risk of exposure of the population to the toxic effects of this molecule.

(C) 2019 International Formulae Group. All rights reserved

Keywords: Foodstuffs, mycotoxins, aflatoxin B1, contamination, exposure.

\section{INTRODUCTION}

Le département de Séguéla, situé dans le Nord-Ouest de la Côte d'Ivoire, regorge d'énormes potentialités culinaires compte tenu de la diversité des produits agricoles rencontrés sur le marché notamment le riz (Oryza sp.), l'arachide (Arachis hypogaea), le gombo (Abelmoschus esculentus), le maïs (Zea mays) et le manioc (Manihot esculenta). Dans cette localité, les denrées sont majoritairement vendues à l'état frais lors des saisons de production; mais lorsque la production excède les capacités d'absorption du marché, le surplus est séché, transformé et conservé (CTA, 2008). Ces denrées transformées sont ensuite utilisées pour de multiples préparations culinaires (Perrin, 2015).

Des études ont montré que le séchage des produits agricoles est un procédé de stabilisation et de conservation. Ce procédé permet de réduire fortement les diverses réactions responsable de la décomposition normale du produit. Cependant, on observe que les denrées séchées sont exposées pour la plupart à une contamination par les moisissures. Les principales moisissures incriminées sont les champignons producteurs de mycotoxines pendant le processus de séchage, de stockage, de manutention et de transport jusqu'à la vente (Mandeel, 2005). Les mycotoxines persistent dans l'aliment même après la cuisson (Bonazzi et Bimbenet, 2003). La contamination des denrées par les mycotoxines affecte aussi bien des produits naturels que transformés tels que les céréales, les oléagineuses, les fruits secs et les produits d'origine animale (Houmairi et Hicham, 2015). Plusieurs mycotoxines parmi lesquelles les aflatoxines, composés hautement cancérogènes et néphrotoxiques ont été répertoriées par les organismes spécialisés comme étant des contaminants des produits agricoles (AFSSA, 2009). Ainsi, en Côte d'Ivoire, des études ont mis en évidence la contamination des denrées alimentaires par les moisissures productrices d'aflatoxines (Boli et al., 2014 ; Dedi et al., 2017). Cependant, peu d'études ont été réalisées sur l'exposition des populations aux moisissures productrices de mycotoxines. Pour renforcer les données relatives à l'exposition des populations aux mycotoxines, l'étude se propose de déterminer les teneurs en aflatoxines des denrées alimentaires de base consommées par la population du département de Séguéla.

\section{MATERIEL ET METHODES}

\section{Présentation de la zone d'étude}

Le département de Séguéla, situé au Nord-Ouest de la Côte d'Ivoire, est le cheflieu région de la région du Worodougou. Il est subdivisé en sept (7) sous-préfectures que sont : Séguéla, Dualla, Kamalo, Sifié, Massala, Worofla, Bobi-Diarabana (Figure 1). La population du département est estimée à 198445 habitants dont 63774 jeunes de moins de 20 ans recensés au niveau de la sous-préfecture de Séguéla (RGPH, 2014). Situé dans la zone de transition entre la forêt et la savane, la localité est propice à l'agriculture. Le département est couvert par un climat de type sud-soudanais avec une saison de pluie allant de juillet à octobre et une saison sèche de novembre à juin. La pluviométrie annuelle moyenne est supérieure à $1200 \mathrm{~mm}$ et la température moyenne est de l'ordre de $28{ }^{\circ} \mathrm{C}$ avec des fluctuations journalières et saisonnières assez marquées (PRICI, 2006). 


\section{Enquêtes alimentaires}

Dans le but de déterminer le régime alimentaire et la fréquence de consommation des aliments, une enquête a été réalisée en octobre 2016 auprès des ménages de la souspréfecture de Séguéla et dans quatre (4) villages (Sualla-Djaminan, Téguéla, Ouahi et Linguekro). Les enquêteurs ont utilisé une balance pèse aliment (CAMRY) et une balance pèse personne (de marque home HBS200-13) pour estimer respectivement les quantités (gramme) de denrées entrant dans la confection des mets et les poids des enquêtés. Une fiche d'enquête comprenant un questionnaire renseignait sur les habitudes de consommation des différents mets, les fréquences de consommation (nombre de fois par jour, par semaine, par mois) sur une durée de un (1) an. L'enquête a été réalisée sur trois cents (300) ménages choisis de façon aléatoire.

\section{Détermination des aflatoxines Echantillonnage}

Un échantillonnage aléatoire a été effectué pour la détermination des aflatoxines dans les denrées de base (riz, pâte d'arachide, farine de maïs et de manioc, poudre de gombo $\mathrm{sec})$. Cet échantillonnage a été mené conformément à l'analyse des mycotoxines selon le règlement $\mathrm{CE} \mathrm{N}^{\circ} 401 / 2006$ du 23 février 2006 et le guide technique d'accréditation LAB GTA 21 révision 02 (2017) du Comité Français d'Accréditation (COFRAC). Ces échantillons de riz, de pâte d'arachide, de farine de maïs, de farine de manioc et de gombo sec moulu ont été prélevés sur le marché de Séguéla où tous les ménages s'approvisionnent. Au total, 100 échantillons à raison de 20 échantillons par matrice ont été prélevés de façon aléatoire sur des sous-lots des différentes denrées de base disponibles sur les étals (Tableau 1). Des échantillons élémentaires ont été pris sur chaque denrée pour constituer un échantillon composite. Les échantillons ont ensuite été conditionnés dans des sachets stomachers et conservés dans une chambre froide à $-20{ }^{\circ} \mathrm{C}$ au laboratoire.

\section{Protocole d'analyse des aflatoxines}

Les aflatoxines ont été analysés selon la norme ISO 16050/2003. Environ vingt (20) $\mathrm{g}$ d'échantillon broyé ont été prélevés et mis dans un ballon de $200 \mathrm{~mL}$. Ensuite, $100 \mathrm{~mL}$ d'un mélange méthanol/eau bidistillée $(80 / 20 ; \mathrm{v} / \mathrm{v})$ y a été ajouté. Le mélange a été homogénéisé à l'aide d'un Ultra-turax (OMNI MIXER) pendant 4 minutes puis centrifugé à $5000 \mathrm{tr} / \mathrm{mn}$ pendant $5 \mathrm{mn}$ à $4^{\circ} \mathrm{C}$ par une centrifugeuse (ZENTRIFUGEN $320 \quad \mathrm{R}$ Hehich). Le surnageant recueilli a été filtré sous vide sur du papier Whattman $\mathrm{N}^{\circ} 4$ dans des erlenmeyers. Dix (10) mL du filtrat ont été prélevés et dilués par $40 \mathrm{~mL}$ d'une solution de tampon PBS (Solution saline de phosphate tamponnée, 10 pastilles/litre d'eau; pH 7,3). Les colonnes d'immunoaffinité AFLAPREP R-Biopharm spécifiques aux aflatoxines ont été sorties du réfrigérateur et laissées au moins 30 minutes à la température ambiante avant leur utilisation. Ces colonnes sont ensuite vidées de leur liquide de conservation et ont été conditionnées avec $10 \mathrm{~mL}$ de tampon PBS à l'aide d'une pompe vacuum à un débit de 1 $\mathrm{mL} / \mathrm{min}$ de façon à ne pas laisser les colonnes séchées. Ensuite, $10 \mathrm{~mL}$ du filtrat ont été prélevés et passés à la purification sur la colonne d'immunoaffinité à un débit de $1 \mathrm{~mL} / \mathrm{min}$. La colonne a été lavée par $10 \mathrm{~mL}$ du même tampon. Les aflatoxines ont été éluées par 1,5 mL de méthanol puis $1,5 \mathrm{~mL}$ d'eau bidistillée y ont été ajoutés au volume recueilli. Un volume de $2,8 \mathrm{~mL}$ est recueilli dans un tube de récupération de $4 \mathrm{~mL}$. Un volume de $20 \mu \mathrm{L}$ d'extrait purifié a été prélevé dans un vial et injecté dans la Chromatographie en phase Liquide à Haute Performance (SHIMADZU Japon) muni d'un détecteur de fluorescence (RF-10AXL). L'acquisition des données a été faite au moyen d'un ordinateur Dell muni d'un intégrateur (logiciel LC Solution). Les concentrations des aflatoxines sont déterminées par la méthode de calcul cidessous:

$$
\mathrm{C}=\frac{\text { Sech } \mathrm{x} \text { Zstd } \mathrm{x} \text { V } \mathrm{x} \text { Vext } \mathrm{x} \text { TR }}{\text { Sstd } \mathrm{x} \mathrm{m} \times \mathrm{Va}}
$$


Où

C: Teneur en Aflatoxine de l'échantillon $(\mu \mathrm{g} / \mathrm{g})$;

standard;

Sech: Surface pic de l'échantillon; $\mathrm{V}$ : Volume du filtrat recueilli (mL);

Zstd : Concentration du Standard $(\mu \mathrm{g} / \mathrm{kg})$; Vext : Volume d'Extraction (mL);

Va: Volume de l'éluât à purifier $(\mathrm{mL})$; $\mathrm{m}$ : masse de l'échantillon (g) ;

TR : Taux de Récupération(\%).

\section{Conditions d'analyse et de validation de la méthode de dosage des aflatoxines à I'HPLC \\ Les conditions d'analyse} chromatographiques sont les suivantes :

- Durée d'analyse : 15 minutes

- Phase mobile : Eau/60 \%; mélange Méthanol-Acétonitrile (v/v : $50: 50) / 40 \%$

- Débit total : $1 \mathrm{~mL} / \mathrm{min}$

- Colonne : C 18 VP-ODS 154 $\mathrm{x} 4.6 \mathrm{~mm}$

- $\mathrm{T}^{\circ} \mathrm{du}$ four $\quad: 40{ }^{\circ} \mathrm{C}$

- Détecteur de fluorescence : RF-10 AXL (365 nm; excitation; 435 émissions)

- Volume injecté : $20 \mu \mathrm{L}$

La détermination quantitative de la teneur en aflatoxines est effectué à travers l'équation de la droite d'étalonnage par rapport à la surface du pic obtenu à la lecture chromatographique. La courbe d'étalonnage représente le rapport de la concentration en fonction de la surface de pic sur une gamme de concentrations d'étalonnage prédéfinie de 0,5 à $20 \mu \mathrm{g} / \mathrm{mL}$.

Condition de validation de la méthode

La méthode a été validée selon les critères de l'Agence fédérale pour la Sécurité de la Chaîne alimentaire notamment la fidélité, la justesse, le taux de récupération, le standard de déviation la Limite de Détection (LD) et la Limite de Quantification (LQ). Les conditions sont résumées dans le Tableau 2.

\section{Estimation de l'exposition à l'AFB1}

L'Apport Journalier Estimé (AJE) en AFB1 est calculé en multipliant les quantités moyennes de denrées consommées par jour par les médianes des teneurs trouvées dans chacune des denrées analysées.

\author{
AJE $(\mu \mathrm{g} / \mathbf{j})=\sum\left(\mathbf{C}_{\text {Med }} \mathbf{X} \mathbf{Q}_{\mathbf{d}}\right)$ (Ricoux et \\ Gasztowtt 2005).
}

Avec :

Cmed: Concentration médiane en AFB1 $(\mu \mathrm{g} / \mathrm{kg})$

Qd: Quantité moyenne de denrée consommée par un individu par jour $(\mathrm{g} / \mathrm{j})$.

Le calcul de Qd prend en compte les quantités moyennes consommées dans les ménages, le nombre moyen de personne dans les ménages et la fréquence de consommation dans l'année.

La Dose Journalière d'Exposition (DJE en $\mu \mathrm{g} / \mathrm{kg}$ de $\mathrm{pc} / \mathrm{j}$ ) est obtenue par la formule de Ricoux et Gasztowtt (2005).

$$
D J E=\frac{\sum A J E}{\mathrm{P}} X \frac{T}{T m}
$$

Avec :

DJE : Dose journalière d'Exposition $(\mu \mathrm{g} / \mathrm{kg}$ de $\mathrm{pc} / \mathrm{j}$ )

AJE: Apport Journalier Estimé en AFB1 $(\mu \mathrm{g} / \mathrm{j})$;

$\mathrm{P}$ : masse corporelle moyen (individus exposés) en $\mathrm{kg}$;

$\mathrm{T}$ : Durée d'exposition moyenne (années) ;

Tm : Période de temps sur laquelle l'exposition est moyennée (années).

Pour les mycotoxines à effets de seuil, les quantités ingérées seront moyennées sur la durée de l'exposition $(\mathrm{Tm}=\mathrm{T})$ et pour les mycotoxines sans effets de seuil, Tm sera assimilé à la durée de la vie entière (prise conventionnellement égale à 70 ans, soit $\mathrm{Tm}=$ 70) (ASTEE, 2003).

\section{Traitement statistique des données}

Les statistiques descriptives et les analyses statistiques ont été effectuées au moyen du logiciel de statistique XLSTAT version 7.5 et le tableur EXCEL 2013. Les données de l'enquête ont été dépouillées et traitées grâce au logiciel statistique ("Le sphinks plus2"). Des recodages ont été effectués sur EXCEL 2013 et les résultats ont été présentés sous forme de tableaux. 


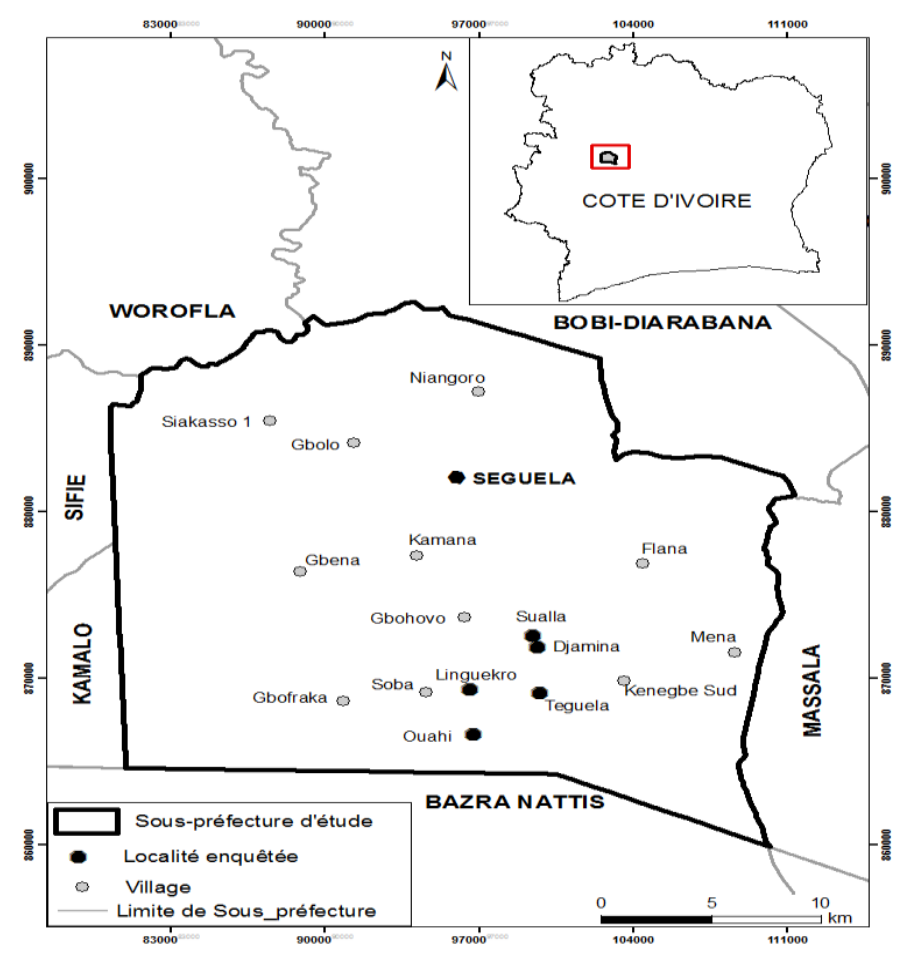

Figure 1: Carte présentant le département de Séguéla (BNETD, 2014 et Enquête, 2016).

Tableau 1: Nombre, types et poids des échantillons prélevés.

\begin{tabular}{lcc}
\hline Denrées prélevées & Masse échantillon $(\mathbf{g})$ & Nombre d'échantillon \\
\hline Riz & 1000 & $\mathrm{n}=20$ \\
Farine de Maïs & 1000 & $\mathrm{n}=20$ \\
Farine de Manioc & 1000 & $\mathrm{n}=20$ \\
Pâte d'Arachide & 500 & $\mathrm{n}=20$ \\
Poudre Gombo sec & 500 & $\mathrm{n}=20$ \\
\hline TOTAL & & 100 \\
\hline
\end{tabular}

Tableau 2 : Conditions de validation de la méthode.

\begin{tabular}{lllllll}
\hline & $\begin{array}{l}\text { Limite } \\
\text { de } \\
\begin{array}{l}\text { Détection } \\
(\boldsymbol{\mu g} / \mathbf{k g})\end{array}\end{array}$ & $\begin{array}{l}\text { Limite de } \\
\text { quantification } \\
(\boldsymbol{\mu g} / \mathbf{k g})\end{array}$ & $\begin{array}{l}\text { Justesse } \\
(\boldsymbol{\%})\end{array}$ & $\begin{array}{l}\text { Fidélité } \\
(\boldsymbol{\%})\end{array}$ & $\begin{array}{l}\text { Taux de } \\
\text { Récupération } \\
(\boldsymbol{\%})\end{array}$ & $\begin{array}{l}\text { Standard } \\
\text { déviation } \\
(\boldsymbol{\%})\end{array}$ \\
\hline AFB1 & 0,00564 & 0,0188 & 6,01 & 1,80 & 93 & 3,01 \\
\hline Critères & & & $\begin{array}{l}\text { Doit être } \\
\text { inférieur } \\
\text { à 6\% }\end{array}$ & $\begin{array}{l}\text { Doit être } \\
\text { inférieur } \\
\text { à 6\% }\end{array}$ & $80 \leq \mathrm{TR}<120$ & $\begin{array}{l}\text { Doit être inférieur } \\
\text { à } 10 \%\end{array}$ \\
\hline
\end{tabular}




\section{RESULTATS \\ Habitude de consommation des denrées et des mets}

L'enquête menée auprès des mères de ménage a permis d'estimer les fréquences de consommation des denrées et les quantités de denrée consommées par jour et par personne. Le Tableau 2 montre les proportions des fréquences de consommation par défaut de plus de 3 fois par semaine et les quantités des denrées de base consommées par jour et par personne au niveau des ménages après les renseignements recueillis grâce au questionnaire de la fiche d'enquête.

L'analyse du tableau montre que les denrées de base des mets consommées de plus de trois fois par semaine sont à des fréquences de $92 \%$ pour le riz, à $42 \%$ pour les farines de maïs et de manioc, à $41 \%$ pour l'arachide et à $38 \%$ pour le gombo sec. L'analyse, des résultats de l'enquête sur la fréquence de consommation des denrées de base et la consommation des mets par les ménages a révélé que le riz cuit, la sauce arachide et la sauce gombo sec sont les mets les plus consommés.

\section{Teneurs en aflatoxines des denrées alimentaires prélevées}

Les résultats d'analyse de l'aflatoxine B1 (AFB1) dans les différents échantillons des denrées alimentaires prélevées, sont indiqués au Tableau 3. L'AFB1, a été détectées dans tous les échantillons analysés. Les teneurs moyennes en AFB1 varient dans les céréales. Pour le riz, elle est de $1,05 \mu \mathrm{g} / \mathrm{kg}$ avec un minimum de $0,01 \mu \mathrm{g} / \mathrm{kg}$ et un maximum de $4,05 \mu \mathrm{g} / \mathrm{kg}$, pour la farine de maïs elle est de $0,92 \mu \mathrm{g} / \mathrm{kg}$ avec un minimum de $0,12 \mu \mathrm{g} / \mathrm{kg}$ et un maximum de $3,18 \mu \mathrm{g} / \mathrm{kg}$. Les teneurs moyennes observées au niveau de la pâte d'arachide et le gombo sec sont supérieures à la teneur maximale tolérable fixée à $2 \mu \mathrm{g} / \mathrm{kg}$ par le règlement $\mathrm{CE} \mathrm{N}^{\circ}$ 1881/2006. Ces teneurs moyennes en AFB1 sont respectivement de $2,38 \mu \mathrm{g} / \mathrm{kg}$ pour la pâte d'arachide et de $2,16 \mu \mathrm{g} / \mathrm{kg}$ pour le gombo sec. La farine de manioc est la denrée la moins contaminée en AFB1 avec une teneur moyenne de $0,67 \mu \mathrm{g} / \mathrm{kg}$ pour des valeurs comprises entre 0,05 et $2,52 \mu \mathrm{g} / \mathrm{kg}$.

Le Tableau 4 indique le nombre d'échantillons pour chaque denrée alimentaire présentant des teneurs en AFB1 supérieures à la norme établie par le règlement dans l'application des normes de sécurité sanitaire des aliments.

Les résultats indiquent que vingt-deux (22) échantillons sur cent (100) échantillons analysés présentent des teneurs en AFB1 supérieures à $2 \mu \mathrm{g} / \mathrm{kg}$, soit $22 \%$ des échantillons sont non conformes à la norme. Il a été observé au travers de ces résultats qu'un nombre élevé d'échantillons de pâte d'arachide (dix (10) sur les 22 échantillons) pourrait être déclaré impropre à la consommation humaine selon la règlementation $\mathrm{CE} \mathrm{N}^{\circ} 1881 / 2006$.

\section{Traitement statistique}

Les tests statistiques ont révélé que $52 \%$ des échantillons ne présentaient pas de différences significatives de contamination en Aflatoxines $(\mathrm{p}<0,05)$ autrement dit, pour ces échantillons la contamination en aflatoxine n'est pas fonction du type de matrice. Par contre, pour $48 \%$ des échantillons, il existe une différence significative entre le type de matrice et la contamination en aflatoxines $(p>0,05)$.

\section{Exposition à l'AFB1}

Le Joint FAO/WHO Expert Committee on Food Additives (JECFA) et le Scientific Committee for Food (SCF) n'ont pas fixé de Dose Journalière Tolérable (DJT) pour les aflatoxines. La seule approche réaliste est de réduire l'exposition à un niveau aussi faible que possible suivant le principe ALARA (As Low As Reasonnably Achievable) (AFSSA, 2009). Cependant, le Conseil Supérieur d'Hygiène Publique de France (CSHPF) a estimé une Dose Journalière Tolérable de 0,15 $\mathrm{ng} / \mathrm{kg} / \mathrm{j}$ pour l'AFB1 et a considéré que, l'ingestion de $1 \mathrm{ng}$ d'aflatoxines/kg p.c./j augmenterait l'incidence du cancer du foie de 0,013 cas par an pour 100000 personnes (AFSSA, 2009). Pour l'AFB1, la durée 
d'exposition (T) a été considérée égale au temps d'exposition sur toute une vie (Tm) en se conférant au seuil fixé. La moyenne des ménages (en ne considérant que les adultes) et le poids moyen des enquêtés estimés lors de l'enquête respectivement de neuf (9) personnes et $64 \mathrm{~kg}$ ont permis de calculer les quantités de denrées consommée par jour, les Apports Journaliers Estimés en AFB1 des denrées et la Dose Journalière d'Exposition (Tableau 6). La Dose Journalière d'Exposition à l'AFB1 liée à l'ingestion des denrées consommées est estimée à $5,6 \mathrm{ng} / \mathrm{kg}$ de $\mathrm{pc} / \mathrm{j}$ avec un apport journalier en AFB1 estimé à $0,36 \mu \mathrm{g} / \mathrm{j}$ en tenant compte des teneurs médianes et des quantités journalières consommées.

Tableau 3 : Consommation des denrées de base dans les ménages.

\begin{tabular}{llllll}
\hline Consommation de Denrée de base & & & & \\
\hline & $\begin{array}{l}\text { Farine de } \\
\text { maïs }\end{array}$ & $\begin{array}{l}\text { Farine de } \\
\text { manioc }\end{array}$ & Riz & Gombo sec & Arachide Pâte \\
\hline $\begin{array}{l}\text { Fréquence de } \\
\text { consommation (\%) (Plus } \\
\text { de } 3 \text { fois par semaine) }\end{array}$ & 42 & 42 & 92,33 & 38,33 & 41,66 \\
\hline $\begin{array}{l}\text { Quantité } \\
\text { consommées/j/pers (g) }\end{array}$ & 68,66 & 94,44 & 242,60 & 8,59 & 75,23 \\
\hline
\end{tabular}

Tableau 4 : Teneurs moyennes en aflatoxine B1 détectées dans les échantillons des différentes denrées alimentaires.

\begin{tabular}{llllll}
\hline \multicolumn{7}{l}{ Teneurs $(\boldsymbol{\mu g} / \mathbf{k g})$ en AFB1 } & & \\
\hline Ech n=20 & Riz & P A & F M C & F M S & P G S \\
\hline Moy & $1,05 \pm 1,13$ & $2,38 \pm 2,22$ & $0,67 \pm 0,65$ & $0,92 \pm 0,99$ & $2,17 \pm 3,95$ \\
Max & 4,05 & 10,25 & 2,52 & 3,18 & 18,65 \\
Min & 0,02 & 0,14 & 0,05 & 0,12 & 0,10 \\
\% Ech. C & 100 & 100 & 100 & 100 & 100
\end{tabular}

Ech : Echantillon ; n=nombre d'échantillon. Moy : Moyenne ; Max : Maximale ; Min : Minimale ; PA : Pâte d'Arachide ; FMC : Farine de Manioc ; FMS : Farine de Maïs ; PGS : Poudre Gombo Sec.

Tableau 5 : Nombre d'échantillons ayant des teneurs supérieurs aux teneurs maximales tolérable en AFB1 et aflatoxines totales.

\begin{tabular}{lll}
\hline $\begin{array}{l}\text { Echantillon de Denrées } \\
\text { alimentaires }\end{array}$ & n & $\begin{array}{l}\text { Nombre } \\
\text { Teneur AFB1 } \mathbf{2} \mathbf{2 \mu g} / \mathbf{k g}\end{array}$ \\
\hline Riz & 20 & 3 \\
Farine de Maïs & 20 & 4 \\
Farine de Manioc & 20 & 1 \\
Pâte d'Arachide & 20 & 10 \\
Poudre Gombo sec & 20 & 4 \\
Total échantillons analysés & 100 & 22 \\
\hline
\end{tabular}


Tableau 6 : Apport et Dose Journalière d'aflatoxine B1.

\begin{tabular}{|c|c|c|c|}
\hline \multirow{2}{*}{ Denrées } & \multirow{2}{*}{ Quantité/j/pers (g) } & \multirow{2}{*}{$\begin{array}{l}\mathrm{C}_{\text {Med }}(\boldsymbol{\mu g} / \mathbf{k g}) \\
\mathrm{AFB} 1\end{array}$} & \multirow{2}{*}{$\begin{array}{l}\mathrm{AJE}(\boldsymbol{\mu} \mathbf{g} / \mathbf{j}) \\
\mathrm{AFB} 1\end{array}$} \\
\hline & & & \\
\hline Riz & 242,60 & 0,55 & 0,13 \\
\hline Arachide & 75,23 & 2,08 & 0,15 \\
\hline Farine de Maïs & 68,66 & 0,43 & 0,03 \\
\hline Farine de Manioc & 94,44 & 0,45 & 0,04 \\
\hline Poudre Gombo sec & 9 & 1,31 & 0,01 \\
\hline$\sum \mathbf{A J E}(\mu \mathrm{g} / \mathbf{j})$ & & & 0,36 \\
\hline DJE $(\mu \mathrm{g} / \mathrm{kg} \mathrm{pc/j})$ & & & 0,0056 \\
\hline DJE (ng/kg pc/j) & & & 5,6 \\
\hline
\end{tabular}

AJE : Apport Journalier Estimé ; DJE : Dose Journalière d'Exposition.

\section{DISCUSSION}

Le riz est la denrée la plus consommé dans le département; selon l'enquête réalisée la majorité du riz consommé est produit localement car la zone est traditionnellement productrice du « riz local». Ces résultats sont en rapport avec ceux de Macauley et Ramadjita (2015) qui ont montré que le riz Ouest africain (Orysa glaberrima) et le riz asiatique (Orysa sativa) constituent la première céréale consommée en Côte d'Ivoire et en Afrique de l'Ouest car c'est la plus importante source d'apports caloriques.

Selon l'enquête, la consommation du riz est accompagnée de diverses sauces, dont la plus consommée dans cette région est la sauce arachide faite à base de pâte d'arachide. Le choix poussé de l'arachide pour les sauces pourrait s'expliquer par ses apports nutritionnels. En effet, il présente des qualités organoleptiques particulières en plus de renfermer une grande teneur en protéines et en lipide (Adjahossou et al., 2009). De plus, l'arachide grillée est riche en antioxydants et en nutriments réputés cardio-protecteurs tel que le resveratrol (Talcott, 2005).

Le tôh est consommé par la majorité des populations autochtones. Comme le département de Séguéla est une zone traditionnellement productrice de manioc et de maïs, les populations se sont adaptées à des mets fait à base des deux denrées. Le maïs et le manioc sont des aliments énergétiques riches en glucide (Zannou et al., 2011). En Côte d'Ivoire, le maïs est la céréale la plus cultivée après le riz surtout dans le Nord et le Nord-Ouest ceci pourrait expliquer logiquement la consommation de cette denrée dans ces zones. (PRESAO, 2011).

Le gombo frais est le plus souvent utilisé comme condiment pour les sauces; mais cette denrée peut être découpée et séchée pour en faire une poudre qui sera utilisée pour la préparation de sauce. Le gombo est beaucoup utilisé dans les repas en Afrique à cause de certaines vertus et des qualités organoleptiques. En effet, ce légume fruit a une valeur nutritionnelle intéressante pour compléter une alimentation déséquilibrée. Il est riche en Fer, Calcium, Cuivre et Zinc. (Kouassi et al., 2013).

Les denrées alimentaires couramment séchées et transformées constituent des cibles non négligeables pour les moisissures toxinogènes notamment l'AFB1 qui est la plus abondante et la plus toxique des aflatoxines (Lahouar, 2016). Il ressort de l'étude que toutes les denrées analysées sont contaminées 
par l'AFB1 et 22\% des échantillons présentent des teneurs en AFB1 supérieures à la norme de $2 \mu \mathrm{g} / \mathrm{kg}$.

Concernant la pâte d'arachide, $50 \%$ des échantillons ont une teneur en AFB1 supérieure à la norme de $2 \mu \mathrm{g} / \mathrm{kg}$. Ces résultats sont en accord avec les études menées par Sangare-Tigori et al. (2006) sur l'arachide produit en Côte d'Ivoire où les échantillons étaient contaminés par l'AFB1 à une teneur moyenne de $4,8 \mu \mathrm{g} / \mathrm{kg}$. De même, les travaux réalisés par Boli et al. (2014) sur les pâtes d'arachides vendues dans neuf communes d'Abidjan ont montré des teneurs en AFB1 allant de 0,23 à 2,49 $\mu \mathrm{g} / \mathrm{kg}$. En effet, l'arachide est sujette à une contamination par les moisissures $\mathrm{du}$ genre Aspergillus productrices de l'AFB1 en Afrique de l'ouest et particulièrement en Côte d'Ivoire (OuattaraSourabie et al., 2001; Boli et al., 2014). Aussi, la contamination des graines d'arachide par les aflatoxines peut avoir lieu dans le sol avant la récolte, pendant et après la récolte au cours du séchage, du stockage et de la conservation (Manizan et al., 2018). Ces facteurs expliqueraient la contamination par l'AFB1 des échantillons de pâte d'arachide constatée dans cette étude. Bien que certaines études aient révélé que la torréfaction des graines d'arachide réduirait la contamination par les aflatoxines, les mauvaises conditions de stockage et de manipulation de la pâte d'arachide pourraient justifier la production de cette mycotoxine (Ogunsanwo and al., 2004).

Le gombo sec est la deuxième denrée la plus contaminée en AFB1 après l'arachide avec une teneur moyenne de $2,16 \mu \mathrm{g} / \mathrm{kg}$. La teneur moyenne dépasse la norme fixée à $2 \mu \mathrm{g} / \mathrm{kg}$ dans les fruits séchés. Les teneurs élevées obtenues dans les échantillons de gombo sec pourraient s'expliquer par une infestation fongique des échantillons de gombo séché. En effet lors de phénomènes tels que le séchage et le stockage, l'affectation de la qualité du gombo et la production d'aflatoxines peuvent être dues à de mauvaises manipulations associées à une mauvaise hygiène (Ouoba et al., 2010; Okigbo, 2017).

Peu d'études ont été menées sur la contamination du gombo sec par les mycotoxines. Toutefois, celles réalisées par Hell et al. (2009) puis par Okigbo (2017) sur des légumes séchés d'Afrique de l'ouest, ont indiqué que des échantillons de gombo séché étaient contaminés par l'AFB1 respectivement de $5,4 \mu \mathrm{g} / \mathrm{kg}$ et $33,490 \mathrm{ppb}$. Ces résultats sont relativement plus élevés que ceux obtenus dans le cadre de notre étude. Pourtant, selon Azzoune (2011), le mode de séchage et la faible teneur en eau des légumes secs réduisaient leur contamination par l'AFB1.

Le riz, les farines de maïs et de manioc sont respectivement contaminés par l'AFB1 à des teneurs moyennes de $1,05 \mu \mathrm{g} / \mathrm{kg}, 0,92$ $\mu \mathrm{g} / \mathrm{kg}$ et $0,66 \mu \mathrm{g} / \mathrm{kg}$. Toutes ces teneurs sont inférieures à la limite de $2 \mu \mathrm{g} / \mathrm{kg}$ fixée par le règlement $\mathrm{CE}$ 1881/2006. La farine de maïs est plus contaminée que celle de manioc. Ces résultats sont en corrélation avec ceux de Matsiko et al. (2017) où des teneurs en AFB1 allant jusqu'à $15,62 \mu \mathrm{g} / \mathrm{kg}$ ont été détectées dans la farine de maïs tandis que de très faible teneurs en AFB1 de 0,15 à $0,5 \mu \mathrm{g} / \mathrm{kg}$ ont été trouvées dans la farine de manioc. Cette différence du niveau de contamination de ces deux types de farine pourrait être due à la variation de la résistance aux champignons des matrices de maïs et de manioc. En effet, une étude menée sur les cossettes de manioc au Bénin, a démontré que l'utilisation in vitro de la scopolétine, une coumarine présente dans le manioc empêcherait la sécrétion des aflatoxines dans les cossettes de manioc (Gnonlonfin et al., 2011). Le manioc est donc un substrat moins favorable au développement des aflatoxines comparativement au maïs. Aussi, la forte contamination de la farine de maïs par rapport à celle de manioc pourrait être due au mode de séchage, de stockage et de transformation du maïs en farine. En effet, le maïs est souvent stocké dans des conditions 
qui ne respectent pas les règles d'hygiènes. Ensuite, pour la transformation, les grains sont préalablement trempés dans l'eau pendant au moins 12 heures avant la mouture. Cette étape de trempage pourrait favoriser le développement de moisissures et la production d'aflatoxines du fait de la teneur en humidité. Selon Nguyen (2007), la teneur en humidité des denrées augmenterait la susceptibilité à une contamination fongique et une production d'aflatoxine. Le riz est une denrée capable d'absorber facilement l'eau de l'air humide ; cette facilité d'absorption d'eau augmente la teneur en eau et donc une susceptibilité à une contamination fongique et une production d'aflatoxine (Nguyen, 2007). Des teneurs en aflatoxine B1 de 1,5 à $10 \mu \mathrm{g} / \mathrm{kg}$ et de 1,5 à $20 \mu \mathrm{g} / \mathrm{kg}$ ont été respectivement trouvées dans le riz et le maïs par SangareTigori et al. (2006). Ces teneurs sont largement supérieures à celles trouvées dans le cadre de notre étude. L'ingestion de denrées contaminées par l'AFB1 expose à une contamination. L'estimation de l'exposition de la population rapportée par l'Apport Journalier de $0,36 \mu \mathrm{g} / \mathrm{j}$ pour toutes les denrées consommées n'est pas négligeable; cet apport correspond à une Dose Journalière d'Exposition de 5,6 ng/kg/j pour un poids corporel moyen de $64 \mathrm{~kg}$. Cette DJE est supérieure à la DJT de $0,15 \mathrm{ng} / \mathrm{kg}$ de $\mathrm{pc} / \mathrm{j}$ fixé par le Conseil Supérieur d'Hygiène Publique de France (EAT, 2004). Cet état de fait indique un risque d'exposition de la population du département de Séguéla consommatrice de ces denrées aux effets cancérogène et hépatotoxique liés à la consommation de denrée contaminées par l'AFB1. En effet, l'exposition chronique à des quantités faibles d'aflatoxine, peut conduire à un risque accru de développer le cancer du foie surtout chez les individus atteint du virus de l'hépatite B (AFSSA, 2009).

Les résultats de cette étude sont confirmés par ceux de Manda et al. (2018), selon lesquelles, l'effet synergique entre l'aflatoxine $\mathrm{B} 1$ et les hépatites virales $\mathrm{B}$ pourrait constituer un facteur de risque important dans l'apparition du carcinome hépatocellulaire en Côte d'Ivoire par la consommation d'aliments à base de denrées telle que le manioc, l'arachide et le riz contaminées par cette toxine.

\section{Conclusion}

La présente étude a permis de déterminer les principales denrées entrant dans la confection de mets couramment consommés par les populations du département de Séguéla. Les principales denrées consommées sont en majorité contaminées par l'aflatoxine B1 à cause des divers procédés tels que le séchage et le stockage généralement effectués dans de mauvaises conditions. La contamination des denrées par l'AFB1 expose la population du département à un risque cancérogène lié à l'ingestion de ces denrées. Mais l'observation des bonnes pratiques de séchage, de stockage et de conservation des denrées aideraient à minimiser la contamination fongique afin de réduire le risque d'exposition des populations à l'AFB1.

\section{CONFLIT D'INTERETS}

Les auteurs déclarent n'avoir aucun conflit d'intérêts en rapport avec les travaux de cet article.

\section{CONTRIBUTIONS DES AUTEURS}

AFD a contribué à l'élaboration du protocole de recherche, à l'interprétation des résultats et à la rédaction du manuscrit. KST a été le responsable scientifique de cette étude. Il a contribué à la validation du protocole de recherche, à la révision du manuscrit et à l'amélioration $\mathrm{du}$ manuscrit. KJMK a supervisé les travaux d'analyse, a participé à la révision et à l'amélioration du manuscrit. $\mathrm{AD}$ a contribué à la valider des protocoles de laboratoire et à la facilitation des travaux 
d'analyse. CAD a contribué plusieurs fois à la révision et à l'amélioration du manuscrit.

\section{REMERCIEMENTS}

Les auteurs remercient le Laboratoire Central d'Agrochimie et d'Ecotoxicologie (LCAE), structure au sein de laquelle les travaux de recherche ont été menés, et qui nous a mis à disposition l'assistance technique.

\section{REFERENCES}

Adjahossou BS, Adjahossou VN, Adjahossou DF, Edorh P, SInsin B, Boko M. 2009. Aspects nutritionnels de l'optimisation d'un système de cultures associant le maïs et l'arachide au Sud-Bénin. International Journal of Biological and Chemical Sciences, 3(5): 1141-1150. DOI:

http://dx.doi.org/10.4314/ijbcs.v3i5.5109 2.

AFSSA (Association Française de Sécurité Sanitaire des Aliments). 2009. Evaluation des risques liés à la présence de mycotoxines dans les chaînes alimentaires humaine et animale. Rapport final. 308p.

ASTEE (Association Scientifique et Technique de l'Eau et de l'Environnement). 2003. Guide pour l'évaluation du risque sanitaire dans l'étude d'impact d'une UIOM. P16

Azzoune N. 2011. Etude des populations du genre Aspergillus et Penicillium et de leur mycotoxines isolées des épices et des légumes secs. Magister Université M'Hamed Bougara de Boumerdès, 110p.

Boli ZA, Zoue LT, Koffi-Nevry R, Koussemon M. 2014. Fungal contamination and mycotoxins occurrence in peanut butters marketed in abidjan district (Côte d'Ivoire). Food and Environment Safety, 13(3): 267-275. http // : www.fia.usv.ro/fiajournal.

Bonazzi C, Bimbenet JJ. (2003). Séchage des produits alimentaires-Principe. Techniques de l'Ingénieur, traité Agroalimentaire, F 3000 1- 14
CTA 2008. Centre de Technique de Coopération Agricole et Rurale. Le séchage des produits agricoles. Postbus 380, 6700 A J Wageningen, Pays Bas disponible sur http://www.cta.int.

Dedi KYJ, Gbehe S, Youo DC. 2017. Caractérisation de neuf échantillons de farine de maïs Zea mays (L.) vendus sur les marchés d'Adjamé, Yopougon et Abobo en Côte d'Ivoire. Journal of Applied Biosciences, 115: 11434-11440. DOI: https://dx.doi.org/10.4314/jab/v115i1.3.

EAT (Etudes de l'Alimentation Totale française) (2004). Mycotoxines, minéraux et éléments traces. p 20.

Gnonlonfin GJB, Adjovi Y, Gbaguidi F, Gbenou J, Katerere D, Brimer L, Sanni A.2011. Scopoletin in cassava products as an inhibitor of aflatoxin production. Journal of Food Safety, 31(4): 553-558. DOI: $\quad$ https://doi.org/10.1111/j.17454565.2011.00334.x

Hell K, Gnonlonfin BGJ, Kodjogbe G, Lamboni Y, Abdourhamane IK. 2009. Mycoflora and occurrence of aflatoxin in dried vegetables in Benin, Mali and Togo, West Africa. International Journal of Food Microbiology, 135: 99104.

http//www.elsevier.com/locate/ijfoodmic ro.

Houmairi H, Hicham M. 2015. Composition en mycobiota et mycotoxines de type aflatoxines et ochratoxine A de quelques épices dans la région centrale du Maroc. Journal of Materials and Environmental Science, $\quad$ 6(3): $\quad 877-884$. http://www.jmaterenvironsci.com/.

ISO 16050/2003. Produits alimentaires. Dosage de l'aflatoxine B1 et détermination de la teneur totale en aflatoxines B1, B2, G1 et G2 dans les céréales, les fruits à coque et les produits dérivés -- Méthode par chromatographie liquide à haute performance.

Kouassi JB, Massara CC, Sess DE, Tiahou GG, Djohan FY.2013. Détermination des teneurs en Magnésium, Potassium, 
Manganèse et Sodium de deux variétés de gombo. Journal of Applied Biosciences, 67:5219-5227. DOI: http://dx.doi.org/10.4314/jab.v67i0.9504 3.

LAB GTA 21 (Guide Technique d'Accréditation) Révision $\quad 02 . \quad 2017$. Dosage des mycotoxines et des phycotoxines dans les denrées alimentaires destinées à l'homme ou aux animaux 23p.

Lahouar A. 2016. Mycotoxines et champignons mycotoxinogènes dans les grains de sorgho commercialisé en Tunisie : Incidence et profils écophysiologiques. Thèse de doctorat 2016 Université de Lleida (Espagne) $225 \mathrm{p}$.

Macauley H, Ramadjita T. 2015. Les cultures céréalières: riz, maïs, millet, sorgho et blé. Centre International de Conférence Abdou Diouf Dakar, Sénégal ; 38 p.

Makeri MU, Bala SM, Kassum AS. 2011. Les effets des températures d'ébullition sur le taux d'extraction et qualité de l'huile traitée localement de deux arachides nigérianes (Arachis hypogea L.) cultivars, African Journal of Food Science, 5: 194-199.

Manda P, Adépo AJB, M'bengue AK, Konan M, N'gbe JV, Doumbia M, Toutou T, Dano SD. 2018. Évaluation du rôle de l'aflatoxine B1 dans l'apparition du carcinome hépatocellulaire en Côte d'Ivoire: étude préliminaire. Toxicologie Analytique \& Clinique, 30(2): 85-95. DOI:

https://doi.org/10.1016/j.toxac.2018.02.0 01.

Mandeel QA. 2005. Fungal contamination of some imported spices. Mycopathologia, 159(2): 291-298. DOI: $10.1007 / \mathrm{s} 11046-$ 004-5496-z.

Manizan AL, Akaki D, Piro-Metayer I, Montet D, Brabet C, Koffi-Nevry R. 2018. Évaluation des pratiques culturales de l'arachide favorisant la contamination par les aflatoxines dans trois régions de Côte d'Ivoire. International Journal of
Biological and Chemical Sciences, 12(4): $\quad 1590-1600 . \quad$ DOI: https://dx.doi.org/10.4314/ijbcs.v12i4.7.

Matsiko F, Kanyange C, Ingabire, G, Dusingizimana T, Vasanthakaalam $\mathrm{H}$, Kimonyo A. 2017. Detection and quantification of aflatoxin in cassava and maize flour sold in Kigali open markets, Rwanda. International Food Research Journal, 24(1): 459-464. http://www.ifrj.upm.edu.my.

Nguyen MT. 2007. Identification des espèces de moisissures, potentiellement productrices de mycotoxines dans le riz commercialise dans cinq provinces de la région centrale du vietnam. Etude des conditions pouvant réduire la production des mycotoxines. Thèse de Doctorat, Institut National Polytechnique de Toulouse, France, 147p.

Ogunsanwo BM, Faboya OO, Idowu OR, Lawal OS, Bankole SA. 2004. Effect of roasting on the aflatoxin contents of Nigerian peanut seeds. African Journal of Biotechnology, 3(9): 451-455. http://www.academicjournals.org/AJB

Okigbo RN, Anene CM. 2017. Prevalence of aflatoxin in dried okra (Abelmoschus esculentus) and tomatoes (Lycoperisicon esculentum) commercialized in Ibadan metropolis. Integrative Food Nutrition and Metabolism, 5(1): 1-4. DOI: 10.15761/IFNM.1000206.

Ouattara-Sourabie PB, Nikiema AP, Traore AS. 2011. Caractérisation de souches d'Aspergillus spp isolées des graines d'arachides cultivées au Burkina Faso, Afrique de l'Ouest. International. Journal of Biological and Chemical Sciences, 5(3): 1232-1249.

Ouoba KH, Desmorieux H, Zougmore F, Naon B. 2010. Caractérisation du séchage convectif du gombo, influence de la découpe et de ses constituants. Afrique SCIENCE, $\mathbf{0 6}(2): \quad 37-48$. http://www.afriquescience.info.

Perrin A. 2015. Étude de la filière Banane Plantain en Côte d'Ivoire. Projet « Promotion et commercialisation de la 
Banane Plantain et du Manioc en Côte d'Ivoire » financé par le Comité Français pour la Solidarité Internationale (CFSI). ONG RONGEAD (International Trade $\&$ Sustainable Development). 66 p.

PRESAO 2011. Programme de Renforcement et de Recherche sur la Sécurité Alimentaire en Afrique de l'Ouest. Rapport Final N ${ }^{\circ}$ 2- 2011-11.

PRICI (Projet de Renaissance des Infrastructures et de gestion urbaine en Côte d'Ivoire) 2006. Financement additionnel au projet d'urgence de renaissance des infrastructures. Financement : Don IDA $n^{\circ} \mathrm{H} 79000-$ CI. Cadre de Gestion Environnementale et Sociale (CGES). Rapport final. 204p.

Règlement (CE) No 1881 / 2006 de la Commission du 19 décembre 2006 portant fixation de teneurs maximales pour certains contaminants dans les denrées alimentaires (Texte présentant de l'intérêt pour l'EEE). Journal Officiel de la Communauté Européenne, L 364 : 05-24.

RGPH (Recensement General de la Population et de l'Habitat de Côte d'Ivoire). 2014. Secrétariat Technique Permanent du Comité Technique du
RGPH. Résultats globaux par régions. p19.

Ricoux C, Gasztowtt B. 2005. Evaluation des risques sanitaires liés à l'exposition de forts consommateurs de produits de la pêche de rivière contaminés par des toxiques de l'environnement.65p.

Sangare-Tigori B, Moukha S, Kouadio J, Betbeder AM, Dano S, Creppy EE. 2006. Co-occurrence of Aflatoxin B1, Fumonisin B1, Ochratoxin A and Zearalenone in cereals and peanuts in Côte d'Ivoire. Food Additives and Contaminants, 23(10): 1000-1007. https://hal.archives-ouvertes.fr/hal00577568.

Talcott ST, Passeretti S, Duncan CE, Gorbet DW. 2005. Polyphenolic content and sensory properties of normal and high oleic acid peanuts. Food Chemistry, 90: 379-388.

Zannou-Tchoko VJ, Bouaffou KGM, Kouame KG, Konan BA. 2011. Etude de la valeur nutritive de farines infantiles à base de manioc et de soja pour enfant en âge de sevrage. Bulletin de la Société Royale des Sciences de Liège, 80: 748-758. 